\title{
Performance Investigation of Signal Strength based Gossip AODV
}

\author{
Prasanna J. Shete \\ K. J. Somaiya College of Engineering \\ Research Scholar, V. J. T. I \\ Mumbai, India
}

\author{
R. N. Awale \\ Dept. of Electrical Engineering \\ V. J. T. I \\ Mumbai, India
}

\begin{abstract}
Due to their "on demand" nature, the reactive protocols for MANETs, incurred less overhead and hence are preferred over proactive protocols. Since reactive protocols rely on network wide flooding of routing messages, they suffer from the "broadcast storm" problem. Received signal strength based probabilistic flooding scheme named RSSGossipAODV was proposed in [13] as an attempt to solve the frequent link breakages and broadcast storm experienced in AODV routing protocol. This paper investigates the ability of RSS-GossipAODV to handle different node mobility speeds in faded and non-faded environments.The performance of RSS-GossipAODV is evaluated by varying node mobility speed. It is evident from our experimental results that in both non faded and Rician faded scenarios, RSS-GossipAODV outperforms conventional AODV in term of packet delivery ratio, throughput, delay and link breakages and at the same time tries to minimize the broadcast storm problem by flooding lesser number of route request (RREQ) messages.
\end{abstract}

\section{General Terms}

Ad Hoc Networks, MANET

\section{Keywords}

AODV, Broadcast storm, RSS, Gossip

\section{INTRODUCTION}

Although the reactive routing protocols $[1,2]$ try to reduce the routing overhead by operating "on demand" to overcome the drawbacks of proactive protocols $[1,3]$, this on demand nature itself leads to another problem. When a data packet is to be delivered to a node for which no path is readily available, the source node discovers it. The route discovery procedure is based on flooding of route request packets (RREQs). The transmission of this special control packet consumes channel bandwidth. No doubt the size of request packet is small, but for a large network with many nodes where destination is many hops away from the source or in case of highly dynamic topology where the routes changes are frequent, this rebroadcast of RREQs by intermediate node (i.e flooding) results in "RREQ storm" in the network [4]. It may happen that in a large network with many nodes, or a small network with high mobility, the entire channel bandwidth is occupied by these RREQs.

Moreover, in case of availability of multiple paths between source and destination, path selection is based on the criteria of shortest path and not the best path. The shortest path may be contributed by weak links. So it may happen that, the discovered path may soon break and become unusable for data transfer and the source has to re-initiate the route discovery. Worst case, route reply (RREP) forwarded on the discovered path may not reach the source! The re-initiation of route discovery procedure in turn worsens the broadcast storm.

To address these issues received signal strength based gossip flooding scheme (RSS-GossipAODV) is proposed. RSSGossipAODV attempts to discover good quality paths in terms of signal strength. Here intermediate node forwards only those RREQs that are received along the hop whose signal strength is above some predefined threshold. For all other, it gossips (probabilistically forwards) with lesser probability.

The rest of paper is organized as follows. Section 2 provides review of similar approaches. RSS-GossipAODV scheme is presented in section 3 its implementation is presented in section 4. Section 5 deals with the simulation setup and performance evaluation. Section 6 concludes the paper.

\section{RELATED WORK}

To optimize the routing mechanism for handling the RREQ broadcast storm problem of reactive protocols various approaches have been suggested. We discuss some of these approaches in this section.

Haas et.al [5] proposed gossiping approach, where each node forwards a packet with some probability. The RREQ broadcasts are controlled by controlling this probability. Modification to AODV named AODV $+\mathrm{G}$ is proposed in which source node sends a route request with probability 1 . When an intermediate node first receives a RREQ, it broadcasts it with a probability $\mathrm{p}$, and discards it with probability $(1-p)$. The gossip based protocol saves up to $33 \%$ messages than flooding, without adversely affecting the network performance. Gossiping significantly improves the performance of denser networks or networks with high mobility, as the numbers of messages generated are quite large and thus percent saving achieved than flooding is significant.

Neighbor coverage based probabilistic rebroadcast protocol (NCPR) for reducing routing overhead is proposed in [6]. This is essentially neighbor knowledge scheme in which each node maintains its 1-hop neighborhood information. Ratio of number of nodes that should be covered by single broadcast to total number of neighbors called Uncovered Neighbor Ratio is calculated to determine how many neighbors should receive the RREQ packet. Based on this "rebroadcast probability" is calculated which is used to reduce the number of rebroadcasts. By combining the neighbor coverage knowledge and probabilistic mechanism the number of rebroadcasts are significantly reduced thus reducing the routing overhead. NCPR generates less rebroadcast traffic than flooding. The performance improvement of NCPR is significant in high density or heavy traffic networks. In sparse networks NCPR performance is slightly better than flooding. However, the algorithm is quite complex. 
Geographic routing approaches use the location coordinates of nodes to forward packets toward the destination in a greedy manner $[7,8]$. By restricting the RREQ flood only in direction of destination rather than network wide, RREQ overhead is reduced. Geographical protocols are scalable since they use localized neighboring information only for next hop selection.

The routing overhead in reactive routing protocols increases due to link failures. To alleviate this limitation a Hybrid Location-based ad hoc routing protocol HLAR is proposed in [9]. HLAR uses the features of greedy geographic routing with reactive protocols and efficiently utilizes the location information to reduce the routing overhead. Also, when the location information is not accurate it uses the basic reactive routing mechanism and avoids the performance degradation. In order to discover a route to destination, the source creates a route request (RREQ) packet that contains location of the source and destination node, it then consults its neighbor table to find if there existed any closer neighbor node towards the destination. If a closer node is available, RREQ is forwarded to that neighbor; if no closer neighbor is available RREQ is flooded to all neighbors. The intermediate nodes follow the same procedure in forwarding the RREQs. The performance evaluation shows that the routing overhead rate of HLAR is constant for various node densities as compared with AODV, in which it grows exponentially. The end-to-end delay is significantly less. Also, the PDR increases as a function of node density because large node density allows for easy route establishment and repair. The performance improvement of HLAR depends on the accuracy of location information. Though provision has been made to reduce the effect of location error, authors point that if this error is large, the performance improvement of HLAR will diminish. This paper does not comment about the effect of overhead arising from inclusion of location information in the RREQ packet.

Estimated Distance-based Routing Protocol (EDRP) is proposed in [10] which restricts the forwarding range of RREQ messages in the direction of destination. EDRP combines the features of position-based routing into ondemand routing protocols. An algorithm is proposed to estimate the distance, called EstD, between two nodes without positioning system. It considers variations in received signal strength (RSS) at contact time of two nodes, to estimate future geometrical distance between them when they move apart. Propagating RREQs in the direction of destination with the help of EstD, significantly reduces the routing overhead and improves the routing performance.

A novel unicast protocol named, Cross Layer Weighted Position-based Routing (CLWPR) designed especially for VANETs in urban environments is proposed in [11]. CLWPR is a position based protocol, which uses the on-road distance as a metric for routing instead of Euclidean distance. Since the nodes are the vehicles travelling along the roads so the onroad distance between nodes will be more accurate than Euclidean distance in the forwarding decision. There is no route discovery phase in CLWPR; rather it relies on 1-hop "Hello" messages that are periodically broadcast by every node. Hello messages carry the position information (position, velocity and heading), MAC frame error rate and size of queue which is used by the nodes to calculate routing table. For each destination that the node has to send a packet it calculates a "weight" of every node in its neighboring list towards that destination. For sending the packets to a specific destination, that node is selected as the next hop node which has the minimum weight. CLWPR demonstrates better performs in terms of packet delivery ratio and end-to-end delay as compared with GPSR. As the "Hello" messages include lots of information, the overhead is significant.

\section{RSS BASED GOSSIP FLOODING}

The strength of wireless transmission decays as a function of distance from the transmitter. Thus the signal power observed at a distance $\mathrm{d}$ from the transmitter can be represented as given in [12].

$$
P_{r}=P_{t} \cdot G_{t} \cdot G_{r} / 4 \pi d^{2}(1)
$$

where, $P_{t}$ and $P_{r}$ represent the transmitted and received signal power, $G_{t}$ and $G_{r}$ are the transmitter and receiver antenna gains, and $\mathrm{d}$ is distance between transmitter and receiver.

For a packet transmitted from sender $\mathrm{x}$ to be successfully received by receiver y the $\operatorname{SINRxy} \geq \operatorname{SINR}_{T h}$.

$$
P_{s}=\operatorname{Prob}\left[\frac{s}{N+I} \geq \beta\right]
$$

where, $\mathrm{s}$ is the received signal strength (RSS), $\mathrm{N}$ is the background noise, $I$ is the interference and $\beta$ is the SINR threshold.

From equation 2 it is clear that successful reception of packet is a function of RSS if we ignore noise and interference. Hence we can rearrange it as

$$
P_{s}=\operatorname{Prob}\left[R S S x y \geq \beta^{\prime}\right](3)
$$

Where $P_{S}$ is the probability of successful packet reception between $\mathrm{x}$ and $\mathrm{y} ; \beta^{\prime}$ is used to represent threshold $\beta$, ignoring noise and interference. In simple terms, it can be stated that two nodes can establish connection if received signal power $P_{r} \geq P_{r T h}$ (i.e $R S S \geq R S S_{T h}$ ).

In reactive protocols route discovery procedure is used to find path to destination for which route is not readily available. It is important that the discovered route should be robust and should experience least failures in case of signal fluctuations. However, the route discovery procedure of AODV doesn't consider the channel quality of hops that contribute to the discovered path. Hence RREQs that arrive on weak or strong links are treated equally. Thus if the discovered path consists of links that are poor, probability of path failure is high. To overcome this issue we proposed a signal strength based gossip flooding mechanism for AODV named RSSGossipAODV [13].

RSS-GossipAODV is essentially a probabilistic flooding mechanism in which RREQ forwarding is based on the signal quality of link along which the request arrives. If the route request was received along a link that is sufficiently strong it is certainlyforwarded, otherwise it is forwarded with lesser probability. RSS-GossipAODV thus attempts to discover robust paths by forwarding more RREQs if they are received on links that offer better signal strength.

RSS-GossipAODV follows Gossip $(p, 1, s)$ approach similar to $\operatorname{Gossip}(p, 1, m)$ suggested in $[5,13]$ where s corresponds to received signal strength threshold $\left(R S S_{T h}\right)$. i.e. Source broadcasts RREQ with probability 1 , and gossiping is used at intermediate nodes only if the RREQ is received along link of poor quality. An intermediate node on receiving RREQ rather than directly rebroadcasting, it checks the RSS. If RSS is above threshold receiving node forwards it with probability 1 , else it gossips with lesser probability. The RSS-GossipAODV algorithm can be summarized as under. 


\section{List1. RSS-GossipAODV Algorithm}

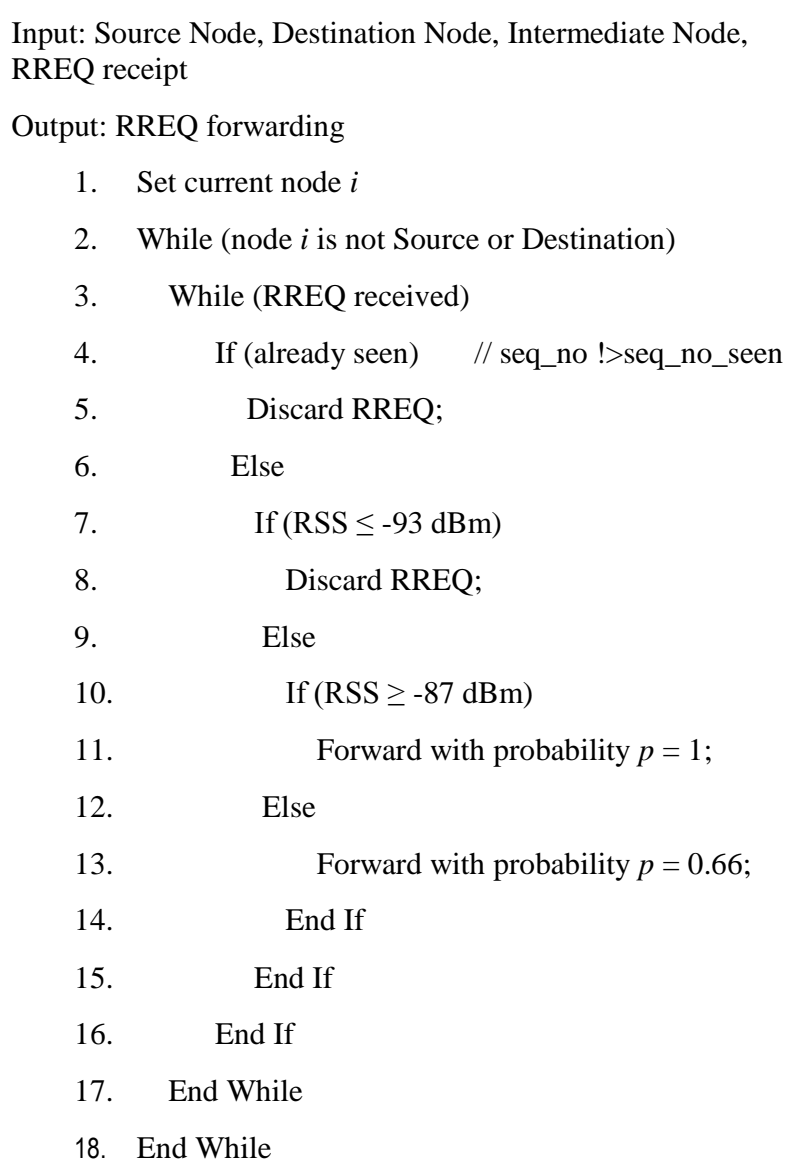

\section{SIMULATION ENVIRONMENT}

RSS-GossipAODV is implemented and analyzed using Qualnet 4.5 simulator. Qulnet's AODV implementation was modified with our proposed scheme and the IEEE 802.11b PHY-MAC of Qualnet was used as lower layer protocols. In our implementation the RSS threshold $\left(R S S_{T h}\right)$ is set as -87 $\mathrm{dBm}$ as it is one of the four receiver sensitivity thresholds used in Qualnet PHY-MAC to decide the MAC sending data rates. The threshold of $-87 \mathrm{dBm}$ in route discovery ensures that good quality paths discovered with certainty will forward data packets at a rate of $5.5 \mathrm{Mbps}$. For weaker links that have RSS lower than threshold will use gossip approach to forward the RREQs and thus avoid network partitioning. Gossip probability is chosen as $p=0.66$ since $p$ value between 0.65 0.75 ensures that almost all nodes receive the messages $\left(\theta_{1}^{S}(0.65)=0.95\right)[5]$.

Performance of RSS-GossipAODV is analyzed by simulating a medium sized ad hoc network consisting of 150 nodes. The nodes are uniformly distributed in a $1000 \mathrm{~m}^{2}$ terrain and move according to random waypoint (RWP) model with minimum speed of $1 \mathrm{~m} / \mathrm{s}$ and pause time of $0 \mathrm{~s}$. $10 \mathrm{CBR}$ traffic connections, each generating traffic at a rate of $4 \mathrm{pkts} / \mathrm{s}$, are set between randomly selected source-destination pairs. Performance is evaluated by varying mobility speed of nodes in non-faded and Rician faded environments. Table 1 summarizes the simulation parameters.

\section{RESULTS}

The performance of RSS-GossipAODV is analyzed on the metrics like packet delivery ratio (PDR), throughput, end-toend delay, link breakages and number of RREQs forwarded.
The results of simulation are plotted and compared with conventional AODV.

In the first experimental scenario we use Two-ray model with no fading environment. Here the node mobility speed is varied from $2.5 \mathrm{~m} / \mathrm{s}$ to $25 \mathrm{~m} / \mathrm{s}$.It is clear from the results (Fig. 1 through 5)that in fading free environments, RSSGossipAODV performs similar to AODV with around 5\% lesser link breakages and 7\% lesser RREQs being forwarded. The link breakages are reduced since the routes discovered are contributed by links that display better signal strength that sustain the varying signal strength arising from changing node speed.

Whereas, in the second scenario when the channel is faded, RSS-GossipAODV outperforms conventional AODV at all speeds. On average, the PDR is improved by $5.5 \%$ and link breakages are reduced by $15 \%$. This is an indicator of the fact that signal strength based routing decision assists to minimize the adverse effects of fading channels. Also, the average number of RREQs forwardedis saved by $15.5 \%$, which in turn reduces the possibility of broadcast storm in the network.

Table 1. Simulation Parameters

\begin{tabular}{|l|l|}
\hline Parameter & Value \\
\hline Number of Nodes and Area & 150 and $1000 \mathrm{~m} * 1000 \mathrm{~m}$ \\
\hline Node Placement Strategy & Uniform \\
\hline Simulation Time & 3 minutes \\
\hline Channel Frequency & $2.4 \mathrm{GHz}$ \\
\hline Path Loss Model & Two ray Model \\
\hline Fading Model & Rician $(\mathrm{k}=0)$ \\
\hline Propagation Limit & $-95 \mathrm{dBm}$ \\
\hline Mobility Model & RWP (pause time $=0 \mathrm{~s})$ \\
\hline Mobility speed (m/s) & $2.5,5.0,10,15,20,25$ \\
\hline PHY / MAC Layer Protocol & IEEE $802.11 \mathrm{~b}$ \\
\hline
\end{tabular}

\section{CONCLUSION}

To address the broadcast storm problem and frequent link breakages experienced in MANETs, signal strength based gossip flooding scheme RSS-GossipAODV, was proposed in [13]. In this work the performance of RSS-GossipAODV in different mobility scenarios is investigated so as to verify its usability in real life scenarios. The performance is analyzed by varying node speeds from $9 \mathrm{~km} / \mathrm{hr}$ to $90 \mathrm{~km} / \mathrm{hr}$, in both faded and non-faded environmental scenarios. It is evident from our experimental results that RSS-GossipAODV performance is superior to conventional AODV in non-faded as well as faded environments. By forwarding $15 \%$ lesser RREQ messages signal strength based gossip flooding approach of RSS-GossipAODV tries to address the broadcast storm problem. At the same time it also improves the network performance by reducing link breakages by $15 \%$ and improving average packet delivery ratio by $5.5 \%$. 


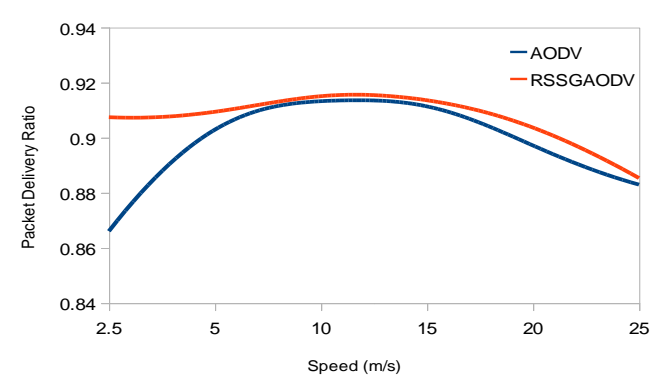

Figure 6. PDR Vs Speed (No Fading)

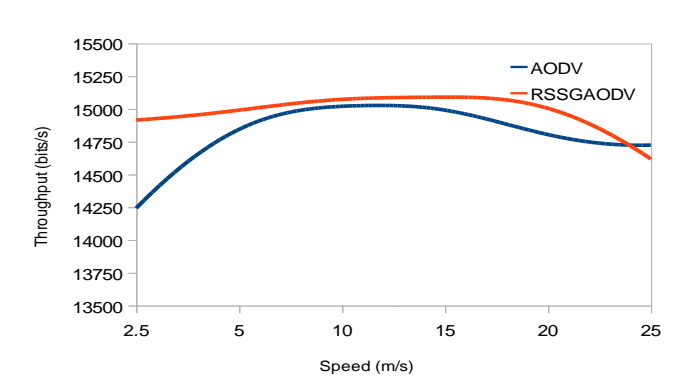

Figure 7. Throughput Vs Speed (No Fading)

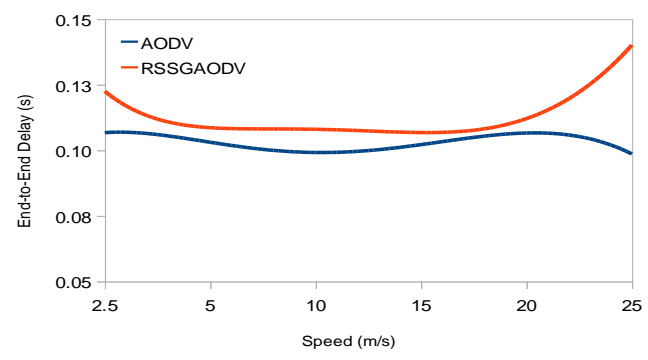

Figure 8. End-to-End Delay Vs Speed (No Fading)

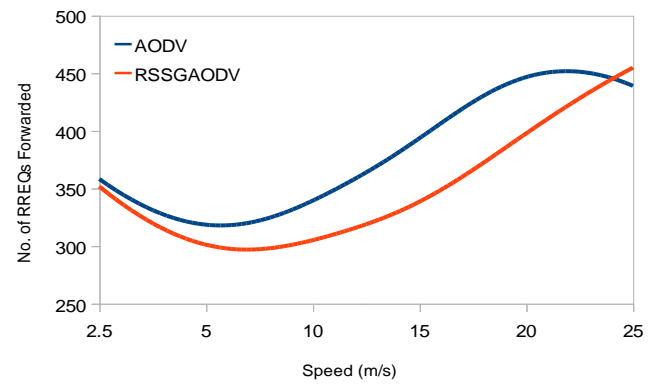

Figure 9. RREQs Forwarded Vs Speed (No Fading)

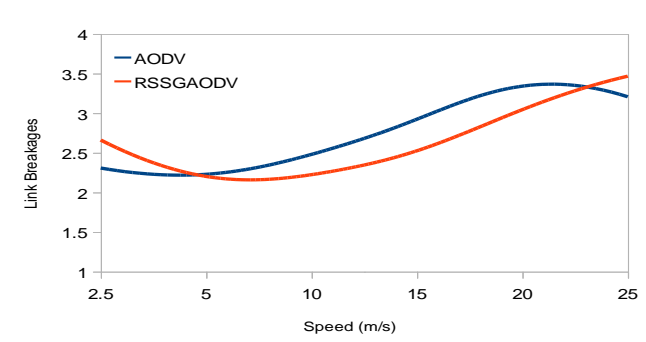

Figure 10. Link Breakages Vs Speed (No Fading) No Fading Statistics

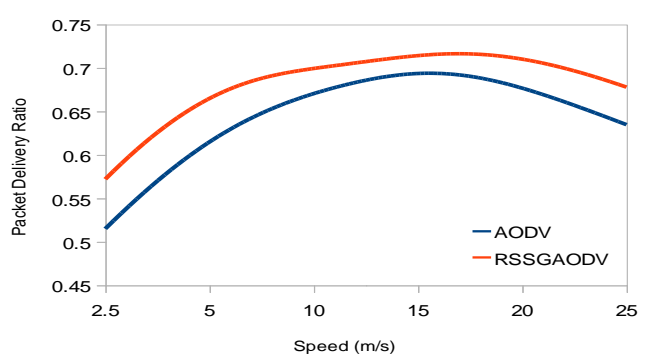

Figure 1. PDR Vs Speed (Rician Fading)

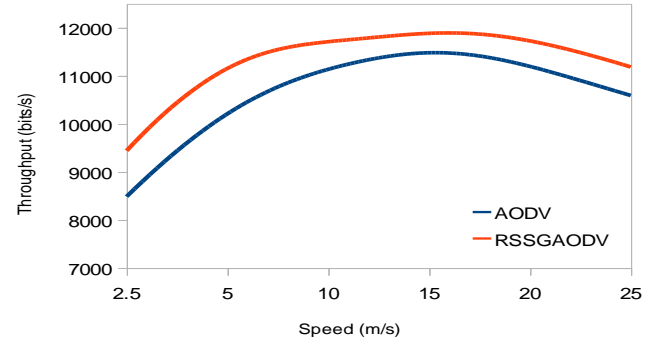

Figure 2. Throughput Vs Speed (Rician Fading)

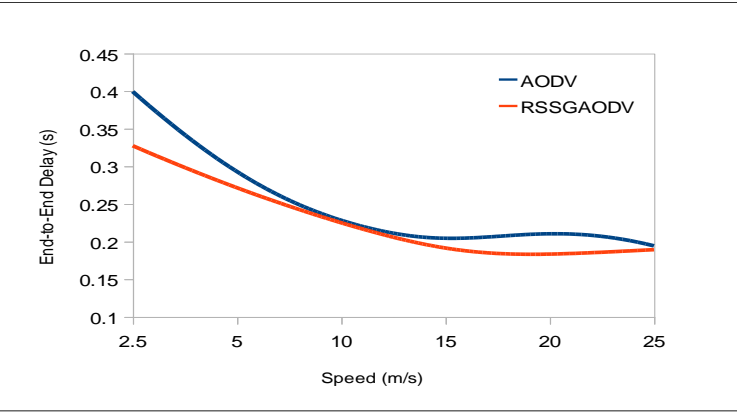

Figure 3. End-to-End Delay Vs Speed (Rician Fading)

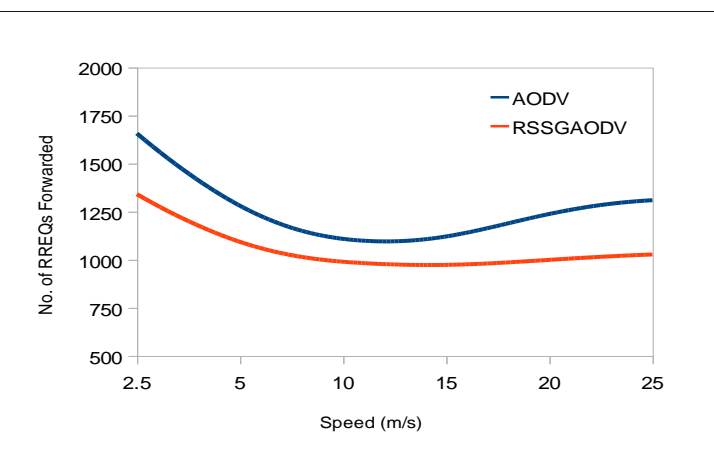

Figure 4. RREQs Forwarded Vs Speed (Rician Fading)

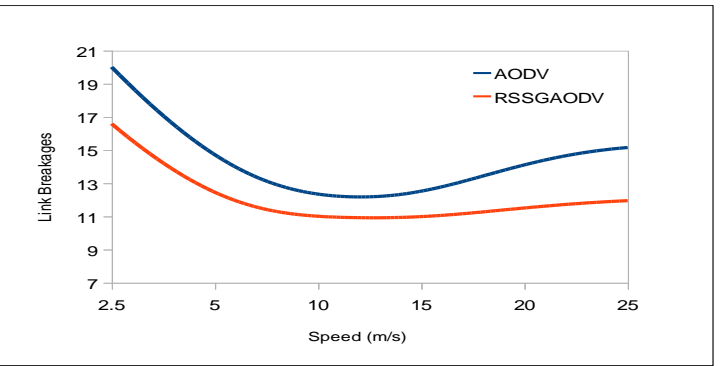

Figure 5. Link Breakages Vs Speed (Rician Fading)

RicianFading Statistics 


\section{REFERENCES}

[1] D. Raychaudhuri and M. Gerla, "Emerging Wireless Technologies and the Future Mobile Internet", Cambridge University Press, 2011.

[2] C. E. Perkins, S. Das "Ad Hoc On-Demand Distance Vector (AODV) Routing”, RFC 3561, July 2003.

[3] C. E. Perkins, P. Bhagwat, "Highly Dynamic Destination-Sequenced Distance Vector Routing (DSDV) for Mobile Computers", Proceedings of ACM SIGCOMM 1994, pp. 234-244, Aug 1994.

[4] O. Tonguz, N. Wisitpongphan, J. Parikh, F. Bai, P. Mudalige and V. Sadekar, "On the Broadcast Storm Problem in Ad hoc Wireless Networks", Proc. of3rd Intl. Conf. on Broadcast Communications, Networks and Systems (BROADNETS), pp.1-11, Oct. 2006

[5] Z. Haas, J. Y. Halpern, and L. Li, "Gossip-based Ad hoc Routing", IEEE/ACM Transactions on Networking, Vol. 14, No. 3, pp.479-491, June 2006.

[6] X. M. Zhang, E.B. Wang, J. J. Xia and D. K. Sung, "A Neighbor Coverage based Probabilistic Rebroadcast for Reducing Routing Overhead in Mobile Ad hoc Networks", IEEE Transactions on Mobile Computing, Vol. 13, No.3, pp. 424-433, March 2013.

[7] Y.B. Ko and N. H. Vaidya, "Location-Aided Routing (LAR) in Mobile Ad hoc Networks", Journal of Wireless Networks, No. 6, pp. 307-321, July 2000.
[8] B. Karp and H. T. Kung, "GPSR: Greedy Perimeter Stateless Routing for Wireless Networks", Proc. of 6th International Conference on Mobile Computing and Networking (MobiCom 2000), August 2000.

[9] M. A Rabayah and R. Malaney, "A New Scalable Hybrid Routing Protocol for VANETs", IEEE Transactions on Vehicular Technology, Vol. 61, No. 6, pp. 2625-2635, July 2012.

[10] X. M. Zhang, E. B. Wang, J. J. Xia, and D. K. Sung, “An Estimated Distance based Routing Protocol for Mobile Ad hoc Networks," IEEE Transactions on Vehicular Technology, Vol.60, No.7, pp. 3473-3484, Sept. 2011.

[11] K. Katsaros, M. Dianati, R, Tafazolli and R. Kernchen, "CLWPR - A Novel Cross-Layer Optimized Position Based Routing Protocol for VANETs", Proceedings of IEEE Vehicular Networking Conference (VNC), pp. 139-146, Nov. 2011

[12] T. S. Rappaport, "Wireless Communication: Principles and Practice", Prentice Hall, 1999.

[13] P. J. Shete, R. N. Awale, "RSS-GossipAODV: Received Signal Strength based Gossip Flooding Mechanism for AODV", Proc. of 6th IBM Collaborative Academia Research Exchange Conference (I-CARE 2014), Oct. 2014. 